Cuadernos de Geografía • $106 \bullet 49-70 \bullet$ València 2021

MARVIN E. QUESADA ${ }^{a}$

\title{
INFLUENCIA DE UNA DEPRESIÓN INTERMONTANA Y LA ALTITUD ENTRE DOS MONTAÑAS EN LA DIFERENCIACIÓN ESPACIAL EN LA PRECIPITACIÓN EN COSTA RICA
}

\begin{abstract}
RESUMEN
Las partes altas de las montañas y sus depresiones se caracterizan por mostrar condiciones climáticas muy distintas comparadas con otras regiones del territorio costarricense. Se investigan cuatro estaciones climáticas: dos ubicadas en las partes altas de la cordillera y dos en un sector de menor altitud, todas coinciden con una depresión intermontana. Esto con el objetivo de visualizar sus diferencias en la pluviosidad entre las estaciones meteorológicas, que se ubican en la dirección en que incursionan las masas de aire por la depresión. Se investigó la precipitación total mensual, el promedio diario mensual, probabilidad de días lluviosos, probabilidad de días con precipitación superior a 5, 10, 20 y 30 mm, y la precipitación máxima diaria mensual. Se ha encontrado que la depresión intermontana que por su condición geomorfológica permite la incursión de masas de aire cargadas de humedad algunos meses del año, lo que contribuye con más humedad la vertiente que normalmente es seca. La precipitación mensual y diaria es más intensa en Miramar y Piedades Sur, mientras que, en cambio, las estaciones de Zarcero y ReBAMB presentan una menor intensidad, pero con más persistencia de precipitación. El hecho de tener estas últimas estaciones una ubicación por encima de los 1500 metros sobre el nivel del mar (m.s.n.m) hace que reciban menor precipitación, dado que el óptimo pluviométrico está por debajo de esa altitud en esa zona del país.
\end{abstract}

Palabras clave: lluvia diaria, masas de aire, montañas, precipitación, ZCIT.

a Investigador y docente Catedrático. Universidad de Costa Rica marvin.quesada@ucr.ac.cr. https://orcid.org/0000-0002-4969-8556

Fecha de recepción: 10/2/2021. Fecha de aceptación: 28/6/2021. 


\title{
INFLUENCE OF AN INTERMONTANE DEPRESSION AND ALTITUDE BETWEEN TWO MOUNTAINS ON SPATIAL DIFERENCIATION IN PRECIPITATION IN COSTA RICA
}

\begin{abstract}
The upper parts of the mountains and their depressions are characterized by difference climatic conditions compared to other regions of Costa Rica territory. Four climatic stations are investigated: two are in the upper parts of the mountain range and two are in a lower altitude sector, all coincide with an intermontane depression. The main point is visualizing their differences in rainfall between the meteorological stations between the upper and lower altitudes, and the roll of the presence of intermontane depression. The total monthly precipitation, the monthly daily average, the probability of rainy days, probability of days with precipitation greater than 5, 10, 20 and $30 \mathrm{~mm}$, and maximum monthly daily precipitation was investigated. The depression has been found to allow the incursion of moisture-laden air masses during some months. which contributes to more moisture in a range that are normally dry. On the other hand, the Stations of Zarcero and ReBAMB have a lower intensity, but with more persistent precipitation. The monthly and daily rainfall is more intense in Miramar and Piedades Sur, while on the other hand, stations like Zarcero and ReBAMB have a lower intensity, but with more persistent precipitation. By contrast, the Zarcero and ReBAMB stations have less amount of rainfall being located at higher altitudes precipitation, The fact of a location above $1500 \mathrm{~m}$ above sea level (m.a.s.l), since the optimal rainfall is below that altitude in that area of the country, which causes them to receive less precipitation.
\end{abstract}

KeYwords: air masses, daily rain, mountain, precipitation, ZCIT.

\section{INTRODUCCIÓN}

La diversidad climática de Costa Rica está altamente controlada por factores geográficos como la latitud, la altitud, la influencia oceánica, entre otros. Todos ellos confieren una diversidad de tipos de climas y microclimas, entre los climas más extremos están los áridos (Guanacaste), hasta los alpinos (Cerro Chirripó). A nivel intermedio existen otros de importancia como son los climas lluviosos, muy lluviosos y pluviales (en este último llueve más y está en un piso altitudinal de mayor altitud con respecto al piso del clima muy lluvioso), entre otros. Entre cada uno de estos climas, se pueden encontrar varios microclimas, que existen dada la diversidad topográfica, altitud y factores como el sotavento y el barlovento.

A pesar de ser un país pequeño, con sólo $51.100 \mathrm{~km}^{2}$, Costa Rica tiene doce zonas de vida en su sector continental, lo que muestra la diversidad de altitud del país (Holdridge, 1982). La irregularidad en el relieve, la influencia marítima, la presencia estacional de la Zona de Convergencia Intertropical (ITCZ), los vientos estacionales y anuales, así como el Anticiclón del Atlántico Norte (AAN), El Niño-La Oscilación del Sur (ENOS) son influenciadores importantes de los regímenes de lluvias del territorio costarricense. 
El elemento del clima más importante en la Zona Intertropical es la precipitación (Waylen, et al., 1996), la cual varía mucho entre las distintas regiones y a cortas distancias. Esto a su vez condiciona la economía de los países del trópico, al estar la mayoría de ellos basados en producción agropecuaria como el café, la caña de azúcar, el banano, los cítricos, hortalizas, tubérculos, frutas y la ganadería, entre otros (Solano y Villalobos, 2016). Además, es muy bien conocido que los países Centroamericanos, desde el punto de vista geológico existe una cordillera que se ubica desde México hasta Panamá que condiciona que existan dos vertientes, la Pacífica y la Caribe. Esto induce a que solo ciertos cultivos se puedan sembrar como por ejemplo banano, algunos tubérculos y la ganadería, condicionando las actividades económicas y las formas de vida de millones de personas (IPCC, 2014).

La vertiente Caribe presenta precipitación durante todo el año, con una única estación: la lluviosa, mientras que la Pacífica presenta dos regímenes de precipitación, con una época lluviosa y otra seca, bien definidas. Más bien en ciertos años se dan intensas sequías, en otras ocasiones inundaciones, en ciertos años se dan algunos meses muy secos o extensión de la estación seca o adelantamiento del período seco. O podría darse, el adelantamiento de la época lluviosa como ocurrió en el año 2021 que dicho inicio se anticipó en abril y no hubo el típico periodo de transición de la época seca a la lluviosa, que normalmente se da en abril.

La variabilidad en la precipitación se debe a patrones de circulación atmosférica que influyen sobre el país y a la irregularidad del relieve. La detección y atribución de cambios en los extremos de lluvia en la escala de tiempo diario (Roy and Balling, 2004; Sohrabi et al., 2013) podría mostrar la frecuencia con la que el tiempo atmosférico cambia en algunas regiones de Costa Rica.

El éxito en la agricultura, la ganadería, y actividades económicas dependen en gran medida de las condiciones atmosféricas y en especial de la precipitación. La falta de precipitación, la extensión de la época lluviosa, los aumentos de días secos en un momento o periodo particular son cruciales en la temporada de cultivos anuales y estacionales, la ganadería y el almacenamiento del agua en fuentes o acuíferos para abastecer la población. Del mismo modo, las lluvias excesivas pueden causar erosión del suelo y la pérdida de semillas y plantas jóvenes, mientras que una secuencia de días húmedos en el momento de la cosecha puede dañar los cultivos o la proliferación de plagas o hongos en los cultivos (Peterson, et al., 2002).

En los últimos años se ha dado una alternabilidad de altas y bajas en la pluviosidad en países como Costa Rica. Desde mediados de 2020 se han observado vientos alisios más intensos en el Pacífico tropical. Así como los vientos del oeste de niveles superiores registrados en esa zona han presentado una intensidad superior a la media (OMM, 2021). Algunos ejemplos de ello son la intensa fase cálida del ENOS en el 2015 y similarmente la fase fría de 2017, 2020, la cual se mantiene hasta abril 2021. Así mismo, existe la influencia de los vientos alisios del noreste (Alisios NE), los cuales soplan con más fuerza (julio y agosto) y Los Nortes con una gran intensidad durante diciembre, enero, febrero e incluso a veces se extienden hasta abril (Poveda et al., 2014).

Además se da la influencia de masas de aire o flujo húmedos denominados la corriente de bajo nivel del Caribe (CBNC), en inglés se le nombra como (Caribbean Low-Level Jet (CLLJ) (Wang, 2007; Hidalgo et al., 2015). Durante el invierno boreal, las incursiones de aire frío (Los Nortes) desde Amé- 
rica del Norte influyen en los vientos y aumento de las precipitaciones que llegan a Costa Rica sobre todo en la vertiente Caribe (Schultz et al., 1998). El propósito de esta investigación es analizar si la precipitación de la región en estudio está determinada principalmente por su posición con respecto a la depresión de El Bajo Tapezco y si existe alguna variación en la pluviosidad con respecto a la altitud por debajo o encima de los 1.500 m.s.n.m., y si existe una contribución de las lluvias diarias provenientes del sector Caribe.

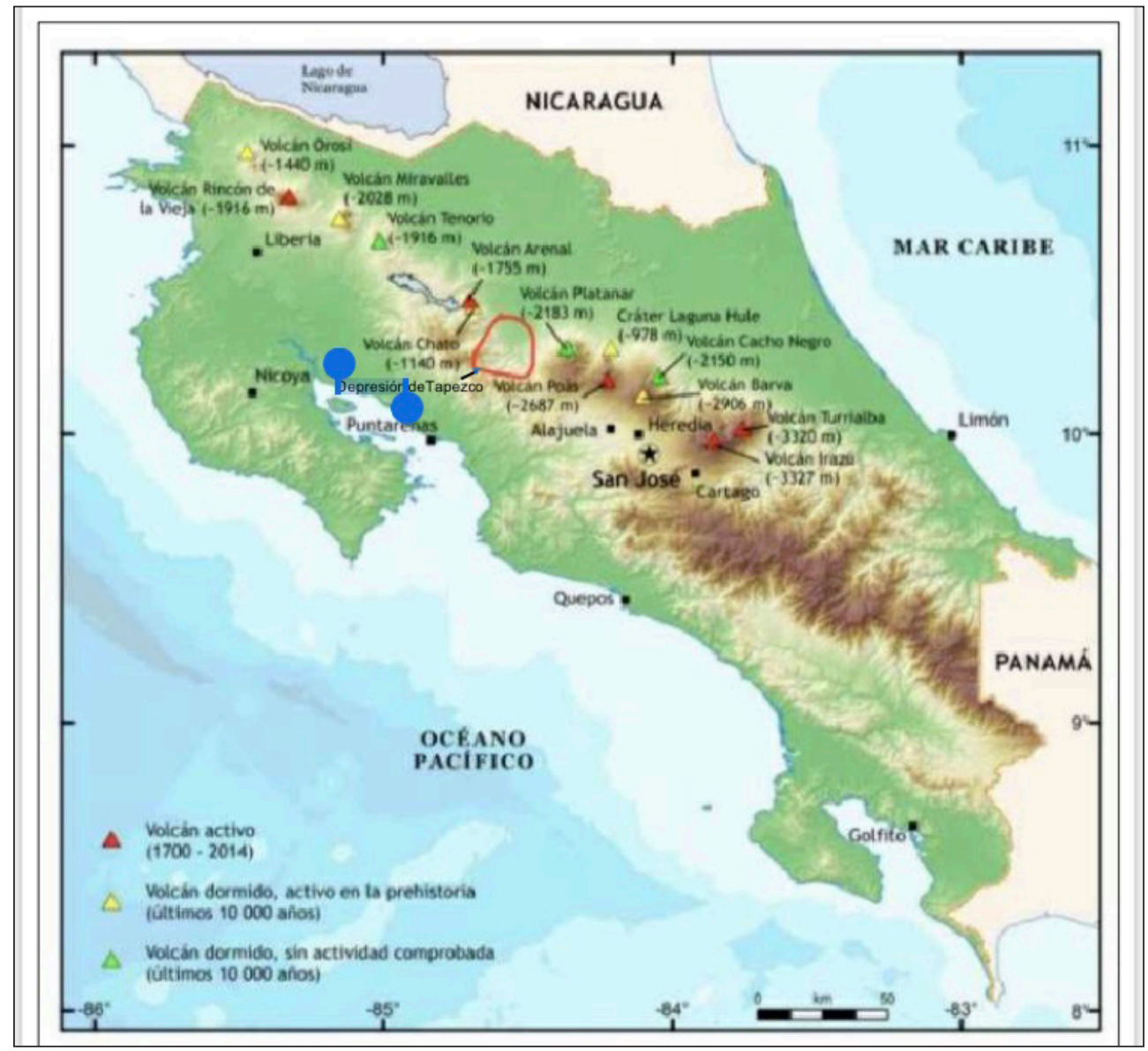

Fig. 1. Ubicación del área en estudio. Depresión del Bajo Tapezco.

Fuente: Instituto Geográfico Nacional de Costa Rica. Ministerio de Transportes. Costa Rica. 2020. 


\section{Área de estudio}

La depresión existente entre las secciones de cordillera de Tilarán y la Central en el Noroeste y parte del centro de Costa Rica es un ejemplo de pasos intermontanos que permiten la entrada de masas de aire cargadas de humedad, como es caso entre la Sección de Tilarán y Central. (figura 1). Esta depresión montañosa recibe el nombre de Bajo Tapezco (Figura 1). Esta depresión intermontana se ubica entre los volcanes Platanar y Póas, siendo muy importante para la región alta, media y baja que es influenciada por la incursión de masas de aire que provienen desde el mar Caribe. Esto permite la agricultura y en especial la horticultura que se desarrolla en la zona alta del cantón de Zarcero. Así como en Piedades Sur en las plantaciones de café, pueda tener suficiente humedad, especialmente durante periodos secos.

Así mismo, los nortes influyen especialmente a finales y a principios de año, que a veces se extiende hasta abril. En otras palabras, esta depresión montañosa presenta condiciones climáticas muy inestables (figura 2). Todas las estaciones meteorológicas que se han considerado en esta investigación tienen cierta influencia de este paso intermontano, unas en mayor grado que otras.

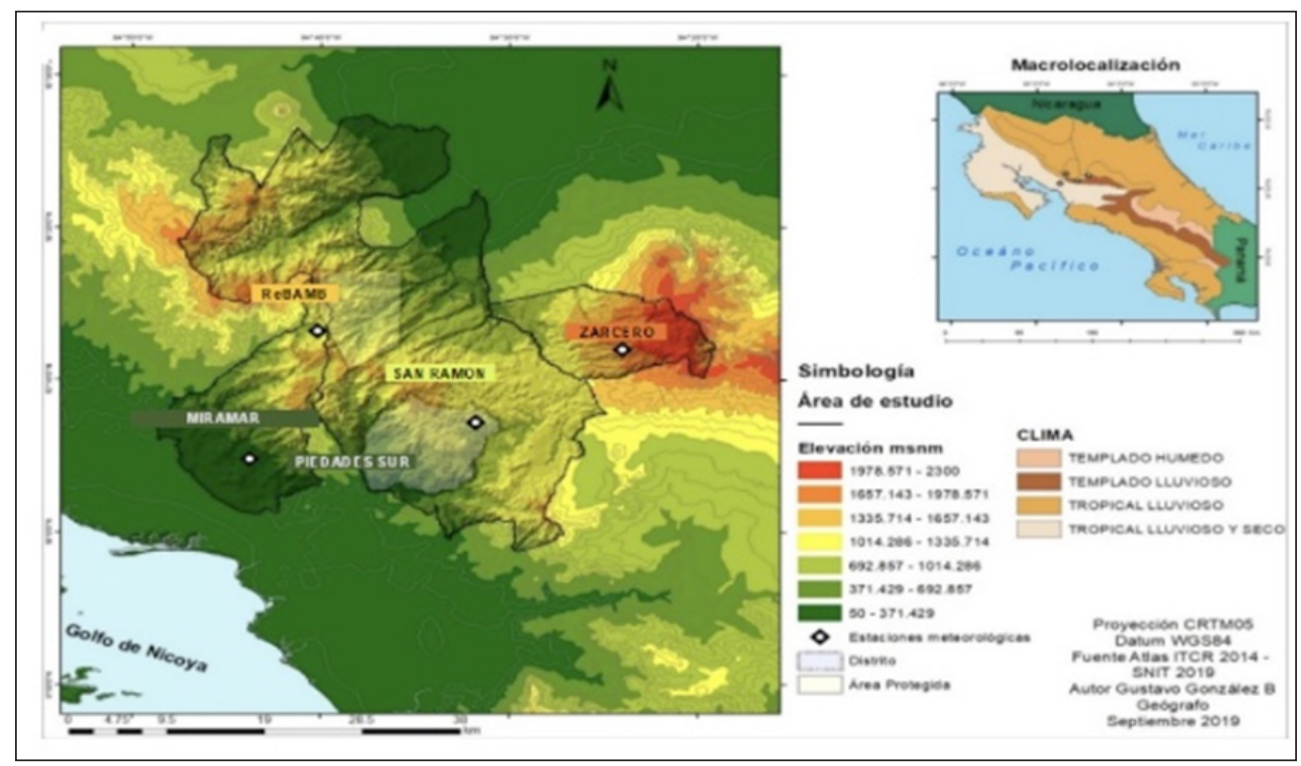

Fig. 2. Ubicación de los sectores o estaciones meteorológicas en estudio.

Fuente: Elaboración propia basada en el Atlas del Instituto Tecnológico Nacional de Costa Rica, 2019. 


\section{DATOS Y MÉTODOS}

El Instituto Meteorológico Nacional (IMN) proporcionó registros diarios digitalizados durante el período 1999-2019 inclusive, o sea se tienen registro de precipitación por un periodo de 31 años. El número de meses de registro completo oscila entre 230 y 240 durante treinta y un años. De cada mes completo de registro, se extraen las siguientes variables: Total de precipitaciones mensuales, las precipitaciones superiores a 5, 10, 20 y $30 \mathrm{~mm}$. De acuerdo con Wang, 2007, se pueden implementar las siguientes ecuaciones con el fin de conocer en la zona en estudio los siguientes aspectos:

\section{- Precipitación diaria mensual}

$$
\frac{1}{m} \sum_{i=1}^{n} P_{i}
$$

Donde $\mathrm{n}=$ Número de días en el mes

Donde $\mathrm{m}=$ número de días en el mes con precipitación medible

- $\underline{\text { Probabilidad de días lluviosos }} \frac{m}{n}$

- $\quad$ robabilidad de tener un total de precipitación diaria superior a $10 \mathrm{~mm}$

$$
\frac{m_{10}}{n}
$$

Donde $\mathrm{m}=$ número de días en el mes con precipitación medible superior a $10 \mathrm{~mm}$ Donde $\mathrm{n}=$ número de días en el mes

- $\quad$ Probabilidad de un total diario superior a $20 \mathrm{~mm}$

$$
\frac{m_{<20}}{n}
$$

Donde $\mathrm{m}=$ número de días en el mes con precipitación medible superior a $20 \mathrm{~mm}$ Donde $\mathrm{n}=$ número de días en el mes

- $\quad$ Probabilidad de un total de precipitación diaria superior a $30 \mathrm{~mm}$

$$
\frac{m_{<30}}{n}
$$

Donde $\mathrm{m}=$ número de días en el mes con precipitación medible superior a $30 \mathrm{~mm}$ Donde $\mathrm{n}=$ número de días en el mes

- Precipitación diaria máxima mensual

$$
\operatorname{Max}(P i) 101
$$


La segunda variable indica la frecuencia con la que se produce la precipitación diaria y de la tercera a la sexta variable se muestra la magnitud de la precipitación desde los 5, 10, 20 hasta $30 \mathrm{~mm}$. El total mensual indica, tanto la frecuencia cambiante como de la magnitud de los eventos.

Con el fin de analizar las lluvias que intervienen por la depresión de El Bajo Tapezco, se contemplaron cuatro estaciones meteorológicas ubicadas en la zona (Zarcero, Reserva Biológica, Alberto Manuel Brenes (ReBAMB), Miramar y Piedades Sur), que tienen al menos treinta y un años de datos completos sin vacíos. Esto es difícil de encontrar en sectores rurales y montañosos, dado que la recolección de estos registros es costosa para las instituciones que se encargan de acopiarlas. Por cierto, en Costa Rica la única forma de adquirir registros meteorológicos es por medio de un contrato oficial que es firmado por el investigador y el director del IMN, dado el alto costo que conlleva la recolección de los registros climáticos sobretodo en regiones montañosas o rurales.

Cuadro 1. Datos de ubicación y total anual de lluvia de las estaciones en investigación. Fuente: Elaboración propia con base en información del Instituto Meteorológico Nacional, 2020.

\begin{tabular}{|l|l|l|l|l|l|}
\hline Número & \multicolumn{1}{|c|}{ Nombre } & Latitud Norte & \multicolumn{1}{|c|}{ Longitud Oeste } & \multicolumn{1}{|c|}{$\begin{array}{c}\text { Altitud } \\
\text { (m.s.n.m.) }\end{array}$} & $\begin{array}{c}\text { Total anual } \\
\mathrm{mm}\end{array}$ \\
\hline 69512 & ZARCERO & $10^{\circ} 11^{\prime} 31^{\prime \prime}$ & $84^{\circ} 23^{\prime} 35^{\prime \prime}$ & 1.736 & 4962,1 \\
\hline 78006 & MIRAMAR & $10^{\circ} 05^{\prime} 36^{\prime \prime}$ & $84^{\circ} 44^{\prime} 05^{\prime \prime}$ & 450 & 7620,8 \\
\hline 80009 & PIEDADES SUR, SR & $10^{\circ} 07^{\prime} 02^{\prime \prime}$ & $84^{\circ} 32^{\prime} 05^{\prime \prime}$ & 1.020 & 7552,4 \\
\hline 78009 & ReBAMB & $10^{\circ} 08^{\prime} 12^{\prime \prime}$ & $84^{\circ} 44^{\prime} 07^{\prime \prime}$ & 1.640 & 4753,8 \\
\hline
\end{tabular}

Las cuatro estaciones meteorológicas tienen la particularidad de tener datos completos durante los treinta y un año de registros y no han sido trasladadas de sitio en ningún momento, desde su instalación. Por consiguiente, es información muy fiable. No obstante, es imperativo señalar que, en países subdesarrollados como Costa Rica, encontrar estaciones meteorológicas en regiones montañosas y muy rurales como la que se investiga es más bien un éxito.

Una de las alternativas de encontrar estaciones medidoras de precipitación en sitios como éstos, es cuando se tiene planeado realizar un proyecto hidroeléctrico, como es la construcción de un embalse. Además, es conocido por los climatólogos a nivel mundial que treinta años es suficiente para realizar un estudio climático. Solamente en aquellos casos que sea una isla se requiere de más años de registro climatológico. Conforme a lo establecido por la Organización Meteorológica Mundial, la normal climatológica o valor normal, se utiliza para definir y comparar el clima y generalmente está representado por el valor promedio de una serie continua de mediciones de una variable climatológica (precipitación, temperatura, viento, etc.) durante un periodo de por lo menos 30 años (IDEAM, 2014). 


\section{Resultados}

El análisis revela que existe una relación significativa entre las características de las masas de aire cargadas de humedad o masas secas con respecto al relieve y los niveles de precipitación en la depresión de Bajo Tapezco. Los efectos que produce la topografía sobre la velocidad y dirección del viento son significativos cuando chocan con una barrera montañosa, ya que se produce una alteración en su distribución. Sin embargo, cuando existe una depresión se produce una canalización, tanto de vientos secos como húmedos y nubosidad, que permiten que lugares o regiones que están en la misma dirección de éstos puedan recibir estacionalmente más o menos precipitación.

Gran parte de la vertiente del Pacífico de Costa Rica experimenta sequías durante unos meses del año diciembre, enero, febrero y marzo. Por lo tanto, la vegetación, la agricultura y la ganadería se ven afectadas por una cierta disminución de las lluvias. Sin embargo, durante los meses de mayo, junio, julio, agosto, septiembre y octubre se producen lluvias como consecuencia de la incursión de los Oestes Ecuatoriales y la ZCIT. Las gráficas de los totales mensuales de precipitación, así como las probabilidades de días con precipitación (figuras 4, 5, 6, 7, 8 y 9), se puede entender claramente que el aumento de los totales mensuales de $\mathrm{M}, \mathrm{J}, \mathrm{J}, \mathrm{A}, \mathrm{S}_{\mathrm{y}} \mathrm{O}$, es el resultado de un aumento tanto en el promedio como en la probabilidad de que llueva más de 5, 10, 20 y $30 \mathrm{~mm}$ diariamente. En tanto, en julio, noviembre y en diciembre se debe a la incursión de los Alisios del Noreste para el primer mes y de los vientos Los Nortes en los restantes meses (figura 3).

La región en estudio muestra la variación en la distribución espacial y temporal en los distintos sectores donde están ubicadas las cuatro estaciones meteorológicas. De ahí que es fundamental el conocimiento de la cantidad de lluvia y la altitud de cada uno de los sectores en estudio.

Las depresiones intermontanas juegan un papel fundamental, dadas las condiciones topográficas, su irregularidad al ser sitios, tanto de lugares cercanos a la línea de crestas como a sitios que se localizan en el pie de monte de las principales secciones montañosas. Al existir una depresión intermontana, eso da pie a que logren pasar masas de aire secas o húmedas, especialmente desde el sector Caribe hasta el Pacífico, lo que conlleva a características climáticas particulares.

Jaramillo (2005) señala como una cordillera sirve de barrera orográfica por su altitud, anchura, longitud y distancia entre barreras vecinas, lo que no permite el paso de masas de aire cargadas de humedad. Así mismo, es imperativo considerar las características de la dirección de las masas de aire y la estabilidad estacional de la atmósfera. Por otro lado, las lluvias están fuertemente controlada por la topografía, alcanzando un máximo a barlovento cerca de la zona donde la pendiente es mayor. Mientras que en la cumbre de las montañas se da una pequeña fracción del máximo de precipitación a barlovento. De ahí que la lluvia aumente con la altitud hasta un nivel máximo que varía según la altitud, que normalmente no sobrepasa los 1.500 m.s.n.m. (Hastenrath, 1991; Ochoa et al., 2016).

Por lo tanto, la lluvia empieza a aumentar desde el fondo de los valles hasta mediados de la montaña o cordillera, siendo necesario que el aire tenga humedad y vapor, factor indispensable que necesita el aire y al enfriarse y al disminuir la humedad con la altitud, deja de haber suficiente condensación para que se produzca la lluvia. De esta forma, el óptimo pluviométrico es la zona donde llueve más y en el 
caso de la zona de estudio es alrededor de los $1.500 \mathrm{~m}$ sobre el nivel del mar. Por encima del óptimo pluviométrico, la disminución de la humedad del aire con la altura y de la cantidad de agua precipitable en las nubes convectivas a escala local son los dos factores que explican la disminución de la precipitación con la altitud (Mendizábal, 1973). En este caso las estaciones meteorológicas de Zarcero y la ReBAMB al estar por encima de los $1.500 \mathrm{~m}$ presentan menos precipitación, que el resto de las estaciones

En la vertiente Caribe y la subvertiente Norte, normalmente se dan condiciones muy diferentes, hay condiciones unimodales, excepto en aquellos casos de estaciones muy cercanas a la divisoria continental o línea de crestas (Jaramillo, 2005). Solamente existe una recesión de lluvias en marzo que es la más baja y otra en octubre, que, a pesar de llamarse disminución, está por encima del valor máximo del sector Pacífico.

Además, dicha depresión montañosa también está influenciada por vientos alisios provenientes del noreste, que influyen sobre la vertiente Caribe y sobre los pasos intermontanos durante gran parte del año. En tanto, a finales y principios de año (diciembre, enero y febrero), entran vientos y masas de aire cargadas de humedad por el norte (Los Nortes) son intensos, fríos y cargados de humedad, ocasionan inundaciones en ciertas regiones del Caribe como Sixaola, Matina, Llanuras Tortuguero, Limón, Sarapiquí y lloviznas constantes en las partes altas de las montañas. Estos vientos están cargados de humedad y generalmente producen precipitaciones en las depresiones intermontanas; cuando son muy intensos persisten desde diciembre hasta abril (Barrantes y Vargas, 2011; Jiménez, 2021).

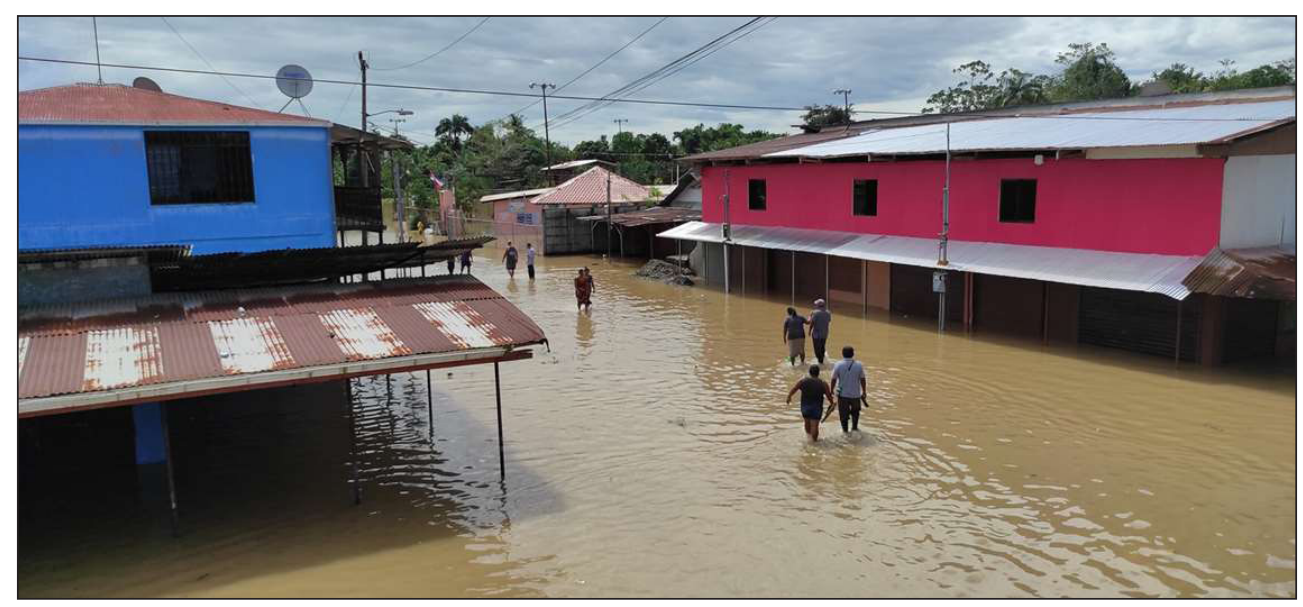

Fig. 3. Inundaciones en Sixaola: "A pesar de que salió el sol, el agua sigue entrando". Limón. Fuente: Jiménez. L. 16-4- 2021. Teletica Canal 7.

Durante el periodo de incursión de frentes fríos, los vientos provienen desde Canadá o Alaska son intensos y en la mayoría de los casos están cargados de humedad. Esto produce intensas lluvias en la parte de la llanura, y en sobre altitudes medias de la vertiente Caribe, donde por lo general se producen 
inundaciones (figura 3) y lloviznas sobre la parte alta de las secciones de cordilleras que tiene Costa Rica. Esto ocasiona la entrada de masas de aire cargadas de humedad sobre las depresiones intermontanas, lo que produce una inestabilidad atmosférica sobre las regiones que están directa o indirectamente en la dirección donde exista una depresión.

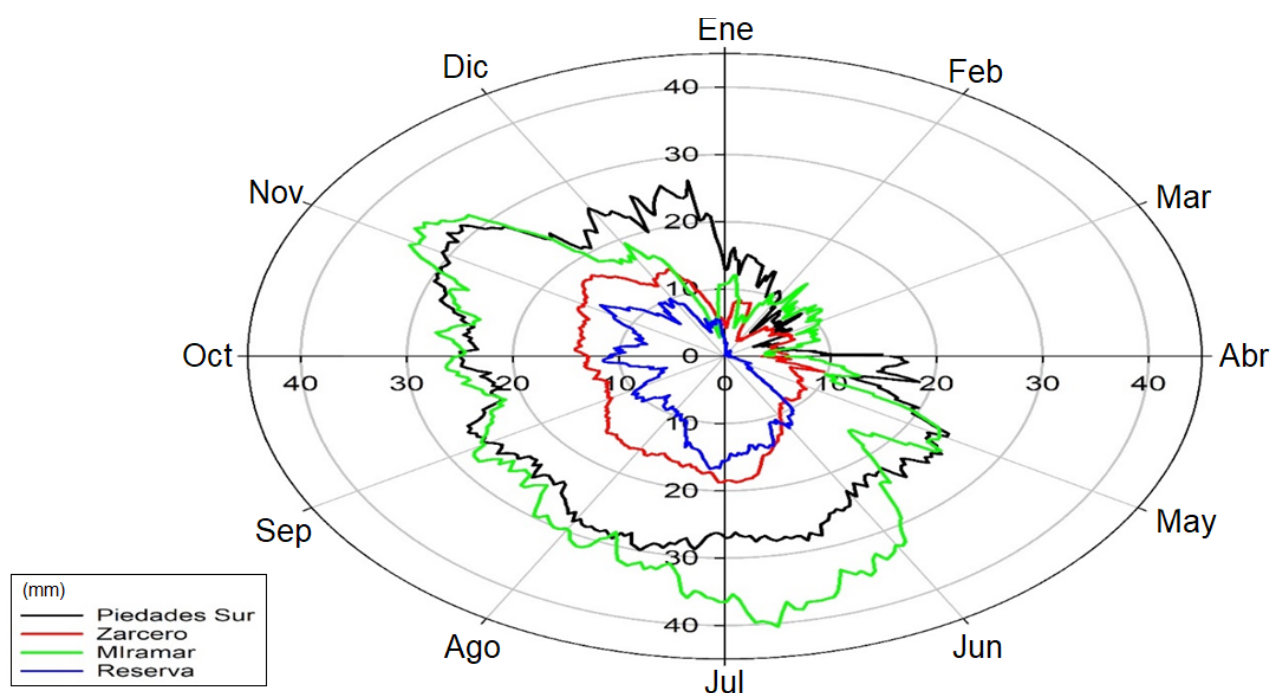

Fig. 4. Promedio mensual de lluvias para Zarcero, ReBAMB, Miramar y Piedades Sur. Fuente: Elaboración propia basada en datos del IMN, Costa Rica, 2020.

En la figura 4 es necesario tener en cuenta los distintos colores para distinguir el comportamiento de la precipitación en cada una de las estaciones meteorológicas en estudio. Tal es el caso de Piedades Sur con color negro, Zarcero en rojo, Miramar en verde y ReBAMB con color azul. El gráfico, aparte de ser muy innovador en climatología, sirve para mostrar en forma circular, iniciando con enero en punto norte o si lo comparamos con un reloj sería el doce, Así sucesivamente, se sigue con el uno, dos hasta llegar hasta a diciembre, que sería las once en un reloj.

Estos son los promedios de precipitación mensual $(\mathrm{mm})$ para las cuatro estaciones en los doce meses del año. Durante diciembre, enero, febrero y marzo se destaca por mostrar una menor cantidad de precipitación. A partir de abril las cuatro estaciones muestran un aumento de las precipitaciones hasta, prácticamente mediados de diciembre. Sin embargo, hay ciertos meses que muestran una mayor cantidad de precipitación, como los meses de mayo a noviembre.

Por otro lado, algunas estaciones meteorológicas muestran una mayor cantidad mensual de precipitaciones, como la estación climática de Miramar y Piedades Sur durante todo el año. Esto se debe a la presencia de ZCIT, que, junto con los vientos los Oestes Ecuatoriales propician dos máximas de precipitación, la primera en mayo y junio y la segunda en septiembre y octubre. Durante los meses de 
julio y agosto disminuyen su velocidad, al migrar al norte la ZCIT, luego de dos meses por lo general regresa hacia la latitud sur y se mantiene cerca de los $10^{\circ}$ latitud $\mathrm{N}$, descargando su humedad.

Durante otros meses como es el caso de noviembre-abril, masas de aire cargadas de humedad del sector caribeño y que logran incursionar a través de la depresión de El Bajo Tapezco, que es el sector de menor altitud de la depresión montañosa que existe entre los tramos de los volcanes Platanar y Póas. Esta condición logra que se mantengan los bosques y la agricultura existente en la parte alta de las secciones montañosas e incluso sectores que se encuentran a una distancia de cerca de $40 \mathrm{~km}$, de distancia como es Miramar y Piedades Sur.

Sin embargo, las estaciones meteorológicas ReBAMB y Zarcero que se encuentran en el lado caribeño tienen menos cantidad de precipitación mensual, siendo la ReBAMB, la que mostró la menor cantidad de precipitación. En estas dos estaciones, pese a la influencia de las masas de aire que se canalizan por El Bajo Tapezco, por su altitud no reciben mucha precipitación, al encontrarse en las cercanías de la divisoria continental.

La mayor parte del sector Pacífico muestra una pequeña disminución en las precipitaciones durante julio y agosto, que se llama veranillos o canículas, que ocurre porque el ZCIT migra hacia una latitud superior a la que se encuentra Costa Rica. La estación de la ReBAMB presenta condiciones de precipitación más similares a la estación Zarcero que, a las otras dos estaciones, dado que ambas están más orientadas al sector caribeño, a pesar de su ubicación muy cerca de la división continental. Mientras tanto, las líneas rojas y azules pertenecen a las estaciones climatológicas de Piedades Sur y Miramar que se ubican en el lado Pacífico, siendo las condiciones de lluvia muy diferentes.

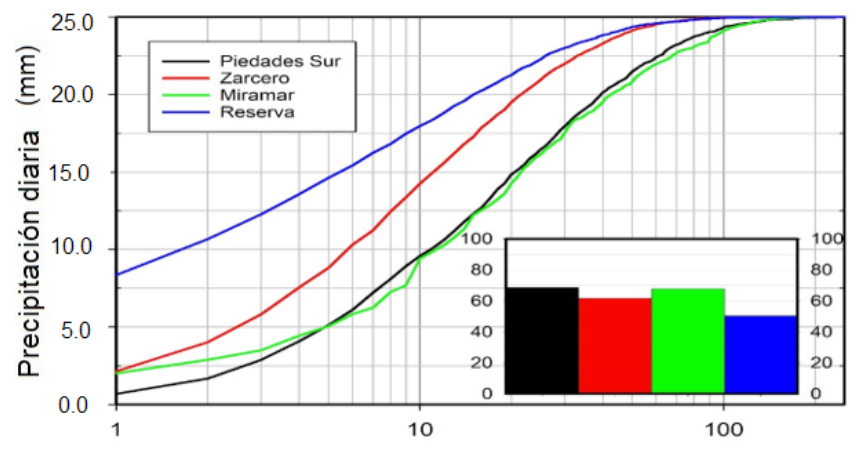

Fig. 5. Precipitación diaria según la frecuencia acumulada en las cuatro estaciones en estudio. Fuente. Elaboración propia basada en registros de estaciones IMN, 2020.

La figura 5 muestra el comportamiento diario de la precipitación para las cuatro estaciones meteorológicas en estudio. En el eje vertical mide la precipitación diaria $(\mathrm{mm})$, mientras que en el eje horizontal se mide la frecuencia acumulada del total de precipitación para las cuatro estaciones. Se pueden ver las similitudes entre Piedades Sur y Miramar. La precipitación que cae en el ReBAMB es diferente 
de las demás, dado que por estar en la vertiente Caribe es más lluvioso, aunque no en su intensidad, se mantiene una lluvia constante y muestra una mayor afinidad con Zarcero.

El pequeño gráfico de barras muestra el porcentaje de todas las observaciones diarias en las cuatro estaciones que no registraron lluvias, tal es el caso de 50 \% de los días en la ReBAMB son lluviosos. Similarmente, existe alrededor del 68 \% de días lluviosos en Piedades y Miramar. Mientras, que en Zarcero muestra un $62 \%$ de la lluvia diaria.

A continuación, se presentan cuatro diagramas que resumen para cada estación en cada mes, los siguientes aspectos: a) La frecuencia relativa de los días secos. b) Las frecuencias relativas de los totales de precipitación que se dividieron en rangos de categoría de $5 \mathrm{~mm}$, hasta $30 \mathrm{~mm}$.

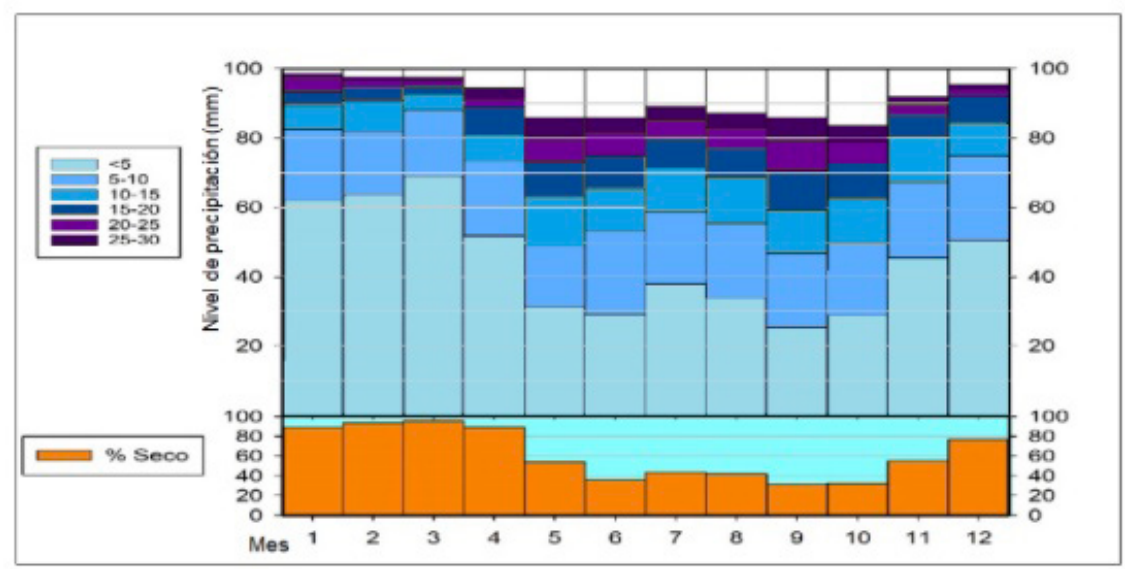

Fig. 6. Distribución mensual como porcentaje de las precipitaciones diarias en Zarcero. Fuente: Elaboración propia basada en datos del IMN, 2020.

La figura 6 muestra la frecuencia de la lluvia durante todo el año en la estación de Zarcero y la magnitud de la lluvia. Este lugar muestra con claridad que desde diciembre hasta abril se presentan meses secos. En tanto, el resto del año con la excepción de julio y agosto que se da una pequeña merma en las precipitaciones se manifiesta que llueve al menos $5 \mathrm{~mm}$. Es así como desde mayo hasta noviembre se tienen valores de lluvia diaria entre 5 y $30 \mathrm{~mm}$ e incluso superiores. 


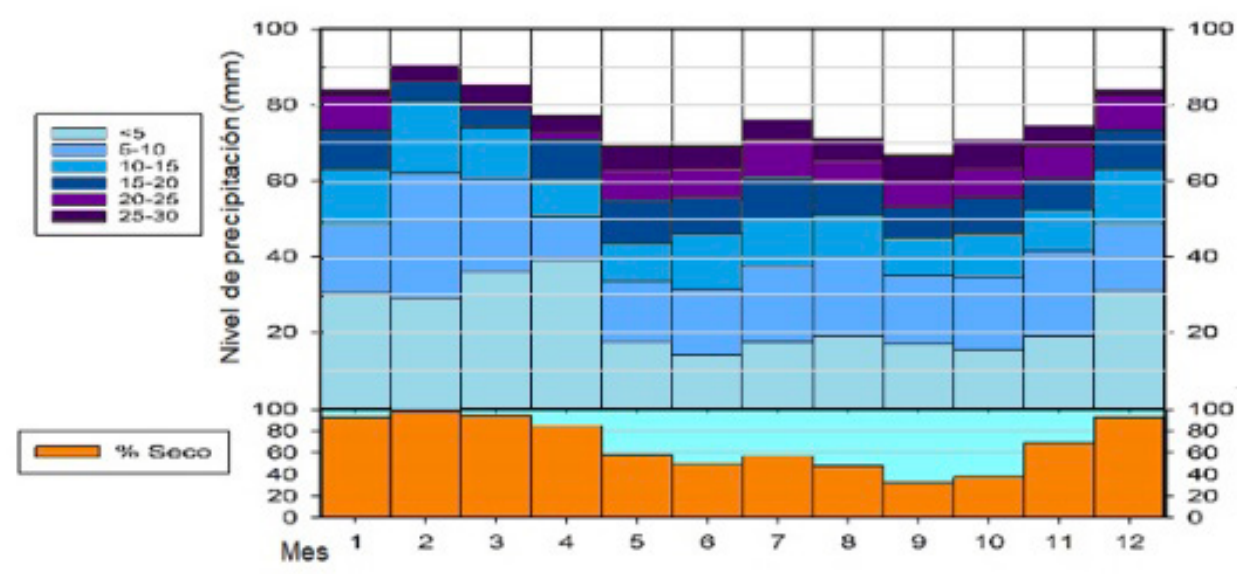

Fig. 7. Distribución mensual como porcentaje de precipitación diaria en ReBAMB. Fuente: Creación propia basada en datos del IMN, 2020.

La figura 7 muestra el porcentaje de todas las observaciones de precipitación diarias, de diciembre a abril se tiene que entre el 90 al $100 \%$ de los días fueron secos. Mientras que entre mayo y noviembre el porcentaje de días secos es de sólo un 40 y 60 \%. En tanto, entre un 15 y un 38 \% muestran días con 5 mm de lluvia diaria. Entre 38 y 80 \% mostraron lluvias entre 10 y $30 \%$ y entre 60 y $80 \%$ mostraron precipitaciones entre 20 y $30 \mathrm{~mm}$. Esta estación muestra una particularidad: el mes más seco es febrero. Aunque los valores de lluvia diaria entre 5 y mayores de $30 \mathrm{~mm}$ son superiores desde diciembre hasta abril, lo que indica que es una estación con mayor influencia de los vientos Los Nortes. En otras palabras, la ubicación con respecto a la depresión intermontana del Bajo Tapezco podría estar influyendo en esos niveles de precipitación diaria en esos meses del año.

La figura 8 se puede observar que la estación de Piedades Sur los meses de diciembre hasta Abril son secos, con porcentajes desde 60 hasta $80 \%$. A partir de mayo-noviembre los meses secos están por debajo del 40 e incluso $10 \%$. Los meses de enero-marzo muestran valores de lluvia superiores al $5 \%$. Asimismo, los meses de abril-noviembre muestran valores entre 50 y $70 \%$ con lluvia de $5 \mathrm{~mm}$. Los meses de mayo-octubre muestran entre 50 y $90 \%$ de lluvias entre 10 y 20 mm. Similarmente, entre un 60 y $90 \%$ presentan valores de lluvia entre 20 y más de 30 mm, entre mayo y noviembre, incluso superiores. 


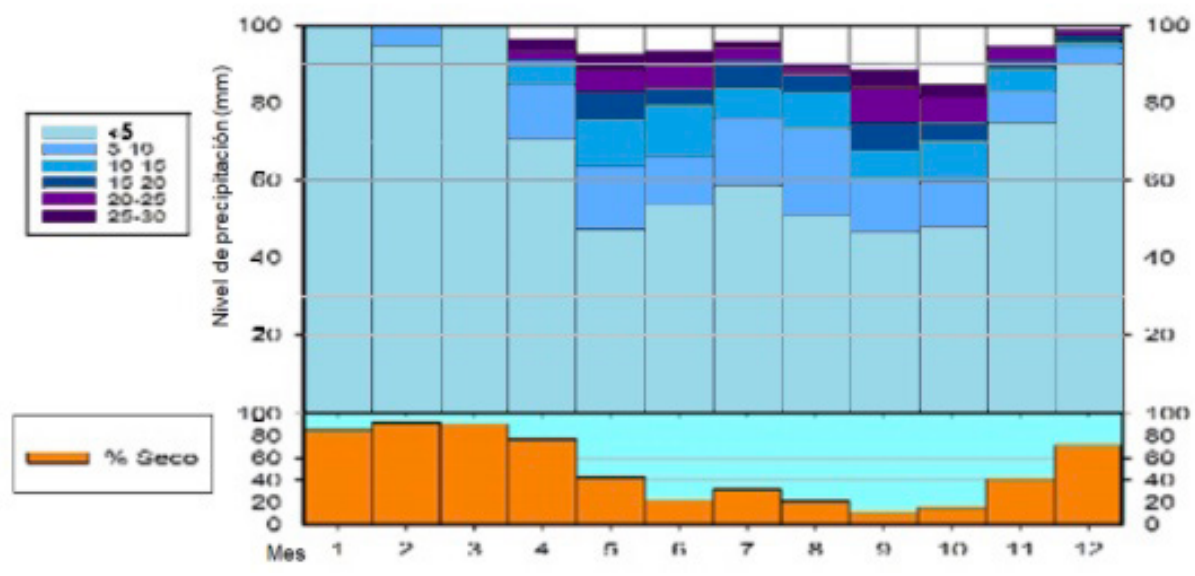

Fig. 8. Distribución mensual como porcentaje de precipitación diaria en Piedades Sur. Fuente: Creación propia basada en datos del IMN, 2020.

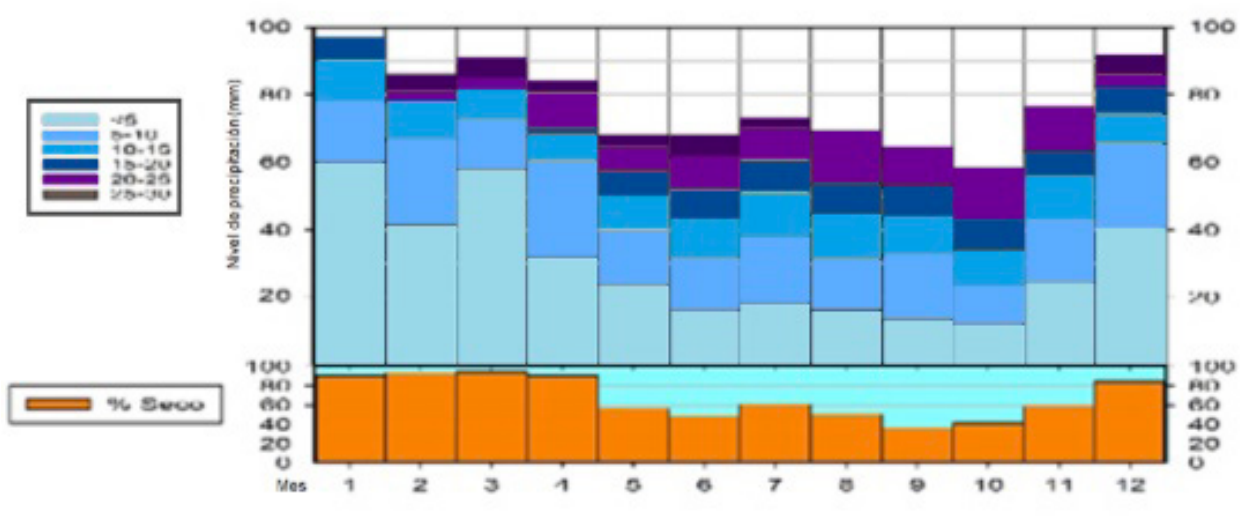

Fig. 9. Distribución mensual como porcentaje de precipitación diaria en Miramar.

Fuente: Elaboración propia basada en datos del IMN, 2020

La figura 9 se muestra que de noviembre a abril los meses secos son menores al $80 \%$ y entre mayo-octubre son menores al $60 \%$. Entre 30 y $60 \%$ presentaron meses con $5 \mathrm{~mm}$ de pluviosidad, asimismo, entre 30 y $80 \%$ con lluvias entre 5 y 10 mm. Es así como entre 20 y $80 \%$ se dan lluvias entre 10 y $20 \mathrm{~mm}$. De igual forma, entre 40 y $90 \%$ se dan precipitaciones entre 15 y $20 \mathrm{~mm}$. Sin embargo, a pesar 
de ser febrero y noviembre meses con altos porcentajes de días secos, se presenta entre un 50 a un $90 \%$ de pluviosidad entre 20 y superior a $30 \mathrm{~mm}$.

Lo anterior se demuestra al observar que, durante los meses secos, la precipitación diaria es de al menos $5 \mathrm{~mm}$; durante estos meses hay aumentos en las precipitaciones superiores a 25 y $30 \mathrm{~mm}$ diarios. Además, se puede observar que es una de las estaciones con menor porcentaje de meses secos. Sin embargo, algo muy interesante ocurre en Miramar, gran parte de los meses mostraron valores entre 25 y $30 \mathrm{~mm}$ de precipitación. En el resto de los meses se tiene que dominan los valores de precipitación diaria entre 10 y $30 \mathrm{~mm}$.

Mientras que las estaciones de Piedades Sur y Miramar muestran una mayor similitud por estar totalmente en el sector pacífico, aunque hay mucha diferencia en la altitud entre una y otra. Para analizar los extremos de precipitación diaria se eligieron dos estaciones, Zarcero con lluvias típicas del piso de mayor altitud y Piedades Sur ubicadas a menor altitud e influenciadas ambas por las masas de aire que ingresan por el sector caribeño. Se encontró que Zarcero presenta una variabilidad en los niveles de precipitación que van desde los 5 hasta los $20 \mathrm{~mm}$. En tanto, en Piedades Sur, existe una predominancia de lluvias de $5 \mathrm{~mm}$ hasta los $30 \mathrm{~mm}$ por día.

Cuadro 2. Datos estadísticos de las lluvias en las estaciones en estudio.

Fuente. Elaboración propia con base en los registros de lluvias del Instituto Meteorológico Nacional de Costa Rica (IMN-CR), 2021.

\begin{tabular}{|l|l|l|l|l|}
\hline \multicolumn{1}{|c|}{ Lugar } & \multicolumn{1}{c|}{ Zarcero } & \multicolumn{1}{c|}{ Miramar } & \multicolumn{1}{c|}{ Piedades Sur } & \multicolumn{1}{c|}{ ReBAMB } \\
\hline Promedio & $1.920,8$ & $2.950,0$ & $2.923,5$ & $1.840,2$ \\
\hline Desviación estándar & 411,0 & 474,6 & 395,8 & 482,3 \\
\hline CV & 0,21 & 0,16 & 0,21 & 0,25 \\
\hline
\end{tabular}

Los datos del cuadro 2 reflejan los datos los análisis diarios de precipitación de cada una de las estaciones analizadas. Como se puede observar existe un mayor porcentaje de precipitación en las estaciones Piedades Sur y Miramar. En tanto, en Zarcero y la ReBAMB los niveles de pluviosidad son menores. Aunque la desviación estándar y el coeficiente de variación son similares entre las cuatro estaciones, los valores más bajos de estos parámetros en la estación de Miramar muestran una distribución más homogénea de la precipitación.

Por la depresión de El Bajo Tapezco incursionan masas de aire húmedo provenientes del Caribe, siendo la causa principal de que estos cambios parezcan ser la intensificación del Jet de Bajo Nivel del Caribe (CBNC), especialmente durante el verano boreal. Esto aumenta la convergencia y las precipitaciones orográficas. Sin embargo, la ligera reversión de este patrón en el invierno boreal, a pesar de un CBNC más intenso, implica una relación más compleja entre la región en estudio y los flujos de aire frío de América del Norte, que son responsables de parte de la lluvia en esa época del año (Hidalgo et al., 2015). 
Las estaciones en estudio se ubican cerca de bosques o cultivos, de ahí que se tenga un microclima particular para cada una de ellas, determinado por las características hídricas y térmicas de su superficie o por su orientación con respecto a los vientos predominantes. Los bosques juegan un papel importante en el balance hídrico de cada estación meteorológica, ya que si están expuestas al flujo de masas de aire reciben mayor pluviosidad y viceversa. Aunque esto no altera en gran medida la cantidad de agua precipitada, sino más bien, altera la forma en que esta es interceptada a través de los árboles, ramas y arbustos y como se desliza por los troncos de los árboles hasta llegar a percolarse en el suelo o escurrir por las superficies que no están protegidas por algún tipo de cultivo o vegetación. Esto ocurre en especial en las estaciones de la ReBAMB y en Miramar. En los otros dos casos de estas regiones de montaña como por ejemplo Zarcero y en Piedades al pie de monte de la sección de cordillera domina la agricultura (figura 10).

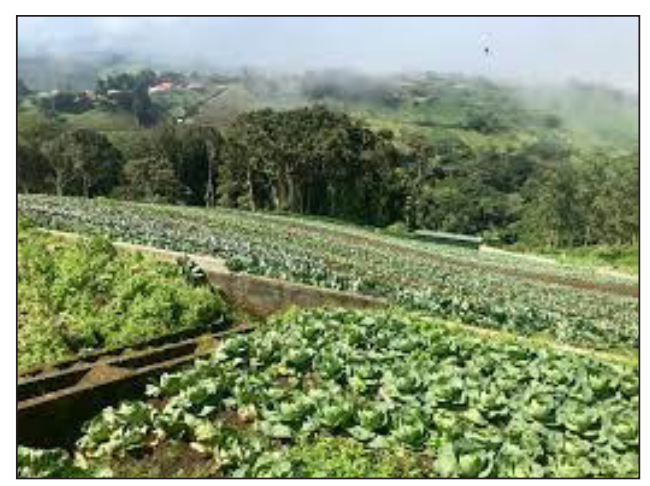

Fig. 10. Agricultura de hortalizas en Zarcero

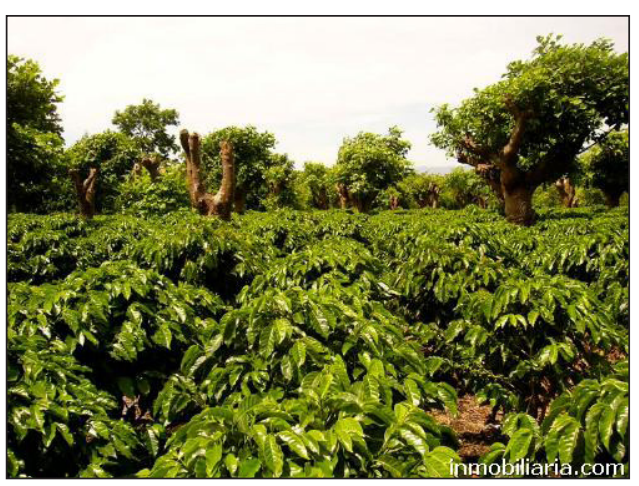

Fig. 11. Caficultura en Piedades Sur, San Ramón

La horticultura necesita suficiente lluvia para poder cosecharse, aunque en exceso puede producir plagas o hongos en las hortalizas. En tanto, la caficultura necesita de una época seca y una lluviosa bien definidas para que el café crezca y madure. El exceso de agua hace que el café se caiga y la poca lluvia provoca que el grano de café no crezca. Sin embargo, si se considera a nivel de mesoescala se podría decir que tanto las estaciones de Zarcero y conforman un microclima característico de los sitios cercanos a la línea de crestas. En tanto, Piedades Sur como Miramar conforman un microclima típico de lugares ubicados a menor altitud pero que también están fuertemente influenciados por las lluvias del Pacífico como del Caribe.

Por el contrario, las estaciones como la ReBAMB y en Miramar, es típico encontrar extensas regiones boscosas. Para el primer caso, al ser una Reserva Biológica, la legislación forestal de Costa Rica sólo permite la visita de investigadores o para fines didácticos y no se permite el turismo, ni ningún otro tipo de actividad que afecte o haga ruido en el bosque. En tanto, Miramar es un lugar montañoso y que recibe muchos turistas durante la mayor parte del año y hay actividades como la ganadería y algunos cultivos. 


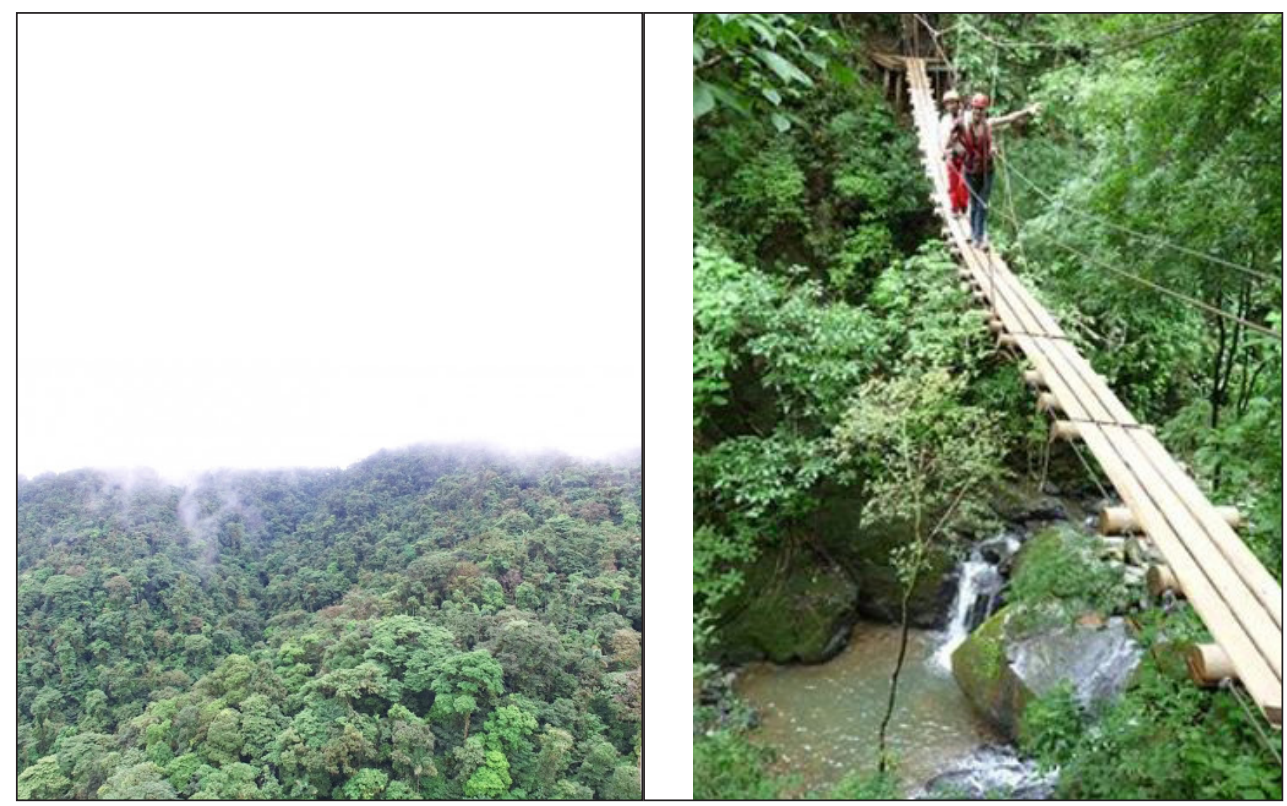

Fig. 12. Bosques de la ReBAMB, San Ramón.

Fig. 13. Bosque y actividad turística en Miramar.

En el caso de la ReBAMB y Miramar, al ser el bosque el principal componente del uso de la tierra, necesitan suficiente lluvia para que los bosques se mantengan siempre verdes o sempervirentes. Sin embargo, en ciertos años se han dado deslizamientos y riadas que han provocado el bloqueo de caminos, carreteras y puentes. En Miramar cuando esto ocurre, el turismo disminuye considerablemente, al no existir paso hacia las áreas boscosas y se pierde la producción agropecuaria y la leche que se obtiene de la ganadería.

Cuadro 3. Población de los lugares investigados (Zarcero, Miramar, Piedades Sur y ReBAMB. Fuente: Estadísticas vitales 2019. INEC-CR

\begin{tabular}{|c|l|l|l|}
\hline Zarcero & \multicolumn{1}{|c|}{ Miramar } & \multicolumn{1}{c|}{ Piedades Sur } & ReBAMB \\
\hline 14.204 habitantes & 14.177 habitantes & 4.450 habitantes & 0 (sólo reserva) \\
\hline
\end{tabular}

En síntesis, se ha demostrado que las estaciones ubicadas en la vertiente Pacífica tienen lluvias más intensas a nivel diario y por consiguiente también muestran influencia de las masas de aire que provienen del sector Caribe, dada su ubicación en la dirección a la depresión de El Bajo Tapezco. Las estaciones ubicadas a altitudes superiores a $1.500 \mathrm{~m}$ tienden a tener lluvia persistente, pero en menor cantidad, son lluvias menos intensas, siendo las estaciones ubicadas en el sector Caribe las que reciben en gran parte las precipitaciones de 5 hasta $30 \mathrm{~mm}$. 
En cambio, las estaciones ubicadas en el sector Pacífico tienen ciertas diferencias. Por ejemplo, Piedades Sur presenta en su mayoría precipitaciones entre los 5 y $30 \mathrm{~mm}$ e incluso más. Mientras que la estación de Miramar presenta lluvias con niveles entre 5 y los $30 \mathrm{~mm}$, pero con la cualidad de que precisamente cuando existen meses secos algunos días del mes presentan valores altos, inclusive de 25 y 30 $\mathrm{mm}$. Miramar y Piedades Sur generalmente tienen lluvias más intensas, pero con menos frecuencia. En la ReBAMB y Zarcero, llueve con mucha más frecuencia, pero sus promedios de lluvias son menores.

\section{DisCUSIÓN Y CONCLUSIONES}

El análisis revela que existen relaciones significativas entre las características de la precipitación diaria y el relieve en la depresión de El Bajo Tapezco y los lugares que se ubican en la trayectoria de influencia de las masas de aire que se canalizan a través de ese paso, hacia la vertiente Pacífica de Costa Rica. Las estaciones ubicadas a menor altitud reciben mayores niveles de precipitación, mientras que a mayor altitud es menor.

Las estaciones de Miramar y Piedades Sur presentan una mayor influencia de la ZCIT, los vientos Alisios, ondas del Este y Tropicales. Por consiguiente, cuando estos ocurren, se pueden dar inundaciones y deslizamientos. Así como pérdidas en la producción agrícola por el exceso de lluvia que causa hongos en las plantaciones o cae la producción agrícola por el exceso de lluvia. Las gráficas de los totales mensuales de precipitación, las frecuencias y probabilidades de días con precipitación (figuras 6, 7, 8,9 ) indican claramente que el aumento es significativo, dado que en las cuatro estaciones los valores de precipitación son superiores a $30 \mathrm{~mm}$.

Los Oestes Ecuatoriales y la presencia de la ZCIT sobre la latitud de la región en estudio hacen que aumenten los niveles de precipitación, dado que se producen constantemente lluvias convectivas, las cuales se producen por lo general durante la tarde, después de que se ha producido un calentamiento durante la mañana en los suelos y la posterior evaporación, lo cual durante las primeras horas de las tardes se forman nubes sobre todo de tipo cumulonimbos que además de intensas precipitaciones, producen tormenta eléctrica y a veces hasta granizos. Mientras que en los meses de noviembre y diciembre mediante la incursión de masas de aire cargados de humedad provenientes del mar Caribe, las regiones a sotavento, como es el caso en especial de Miramar y Piedades Sur, reciben ciertos niveles de precipitación que van entre 10 a 30 mm e incluso más por día.

El patrón espacial climático mostrado por las cuatro estaciones en estudio varía según la ubicación e intervienen factores como el relieve que condiciona mayor o menor precipitación diaria entre los valores de 5 y $30 \mathrm{~mm}$, en una u otra estación. Sin embargo, hay cierta similitud como ya se ha mencionado entre las estaciones ubicadas en las inmediaciones de la divisoria continental como Zarcero y la ReBAMB, aunque con diferentes niveles de precipitación.

Durante el invierno boreal, las incursiones de aire frío (Los Nortes) de América del Norte están asociadas a la precipitación y la convergencia superficial (Schultz et al., 1998). Tanto los Alisios como Los Nortes fluctúan en mayor o menor medida con las fases de ENOS (Waylen et al., 1996; Poveda et al., 2006). Por lo tanto es razonable pensar que esto se reflejará en las características de las precipitacio- 
nes diarias, que tienen una gran importancia local para la agricultura y la planificación de los recursos hídricos (Quesada and Waylen, 2020).

Desde el punto de vista del microclima, las condiciones de tiempo atmosférico son modificadas por las locales, tales como la presencia de coberturas boscosas, cultivos, ríos y la topografía. Si fuera posible tener estaciones más cercanas una de otras o un mayor número de estaciones meteorológicas se podrían encontrar una mayor variedad de microclimas. Pero como ya se dijo, en este tipo de regiones montañosas y rurales es muy difícil la presencia de estaciones a corta distancia, lo cual es muy típico en los países pobres del trópico.

Uno de los factores más relevantes es la distribución espacial de la precipitación, al darse diferentes niveles de lluvia en cimas, pendientes, valles de la región en estudio, se da producto de la interacción entre la topografía y las masas de aire. Esto se demuestra al tenerse que las estaciones ubicadas a mayor altitud reciben niveles constantes de precipitación, pero las regiones ubicadas a menor altitud tienen más intensidad en las lluvias. Las estaciones de Miramar y Piedades Sur se presentan condiciones mixtas en cuanto al origen de las precipitaciones, o sea reciben lluvias provenientes, tanto del océano Pacífico como del mar Caribe.

Además, la concavidad entre los dos volcanes (Platanar y Póas), crea condiciones favorables para el paso de masas de aire cargadas de humedad que contribuyen con precipitaciones en periodos que normalmente son secos en la vertiente Pacífica. De ahí que la incursión de humedad desde el sector Caribe se da principalmente en los meses de junio, noviembre, diciembre, enero y febrero, que precisamente es cuando se da el periodo seco en la vertiente donde se ubican estas dos estaciones. Esto permite que lleguen precipitaciones y permitan el crecimiento del bosque, cultivos, ganadería y turismo, sin la necesidad de usar riego y regiones como Miramar y Piedades Sur y así poder mantener sus ingresos económicos.

\section{Agradecimientos}

Se reconoce el apoyo de la Vicerrectoría de Investigación por el financiamiento parcial del estudio, así como la coordinación de Investigación de la Sede de Occidente por permitirme llevar a cabo el presente estudio. Ambos pertenecen a la Universidad de Costa Rica. Además, un agradecimiento muy particular al Instituto Meteorológico Nacional de Costa Rica por confiar en mi persona y suministrar los datos de las cuatro estaciones meteorológicas.

\section{ReFERENCIAS}

Barnard, P.L. et al. (2015): Coastal vulnerability across the Pacific dominated by El Nino/Southern Oscillation. Nature Geosciences, 8: 801-807.

Barrantes, G y J. Vargas (2011). La Zonificación de amenaza por inundación como herramienta para el ordenamiento territorial en el Valle del Río Sixaola. Revista Geografía de América Central, 46: pp. 67-85. 
Cai, W. et. al. (2014). Increasing frequency of extreme El Niño events due to greenhouse warming. Nature Climate Change, 4(2), pp.111-116.

Costa Rica. Comisión Nacional de Prevención de Riesgos y Atención de Emergencias. Estrategia Nacional de Contingencia para Enfrentar los Efectos de El Niño en Costa Rica, período 2014-2015 / La Comisión. Unidad de Desarrollo Estratégico del SNGR y Carlos Picado Rojas. - San José, C.R. CNE, 2015: 32 p.

Enfield, D.B. and Alfaro, E.J., (1999). The dependence of Caribbean rainfall on the interaction of the tropical Atlantic and Pacific oceans. J. Climate 12, 2093-2103

Enfield, D., Mesta-Nunez, A., Markgraf, V. (Eds.), (2001). Interhemispheric Climatic Linkages. Academic Press, San Diego. 454 pp.

IPCC (2014). Summary for policymakers: Climate Change. Impacts, Adaptation, and Vulnerability. Part A: Global and Sectoral Aspects. Contribution of Working Group II to the Fifth Assessment Report of the Intergovernmental Panel on Climate Change ed. CB Field et al. (Cambridge) (Cambridge University Press) (Cambridge, United Kingdom and New York, NY, USA) pp 1-32.

Glantz, M.H., (2001). Current of Change: Impacts of El Nino and La Nina on Climate and Society, 760 pp. Cambridge University Press, Cambridge, U.K.

Guillen-Oviedo, H.S., Cid-Serrano, L.R. and Alfaro-Martínez, E.J., (2020). Comparación de parámetros de valor extremo de la distribución generalizada asociada a eventos de precipitación extrema en América Central. Uniciencia, 34, 1: pp. 111-128

Hastenrath, S. (1991). Climate dynamics of the tropics. Boston: Kluwer Academic Publ.

Hastenrath, S., (1998). Contribution to the circulation climatology of the eastern equatorial Pacific: Lower atmospheric jets. J. Geophys. Res. 103 (D16), pp. 19443-19451.

Hidalgo, H.G., Durán-Quesada, A.M., Amador, J.A. and Alfaro, E.J., (2015). The Caribbean low-level jet, the inter-tropical convergence zone and precipitation patterns in the intra-Americas Sea: A proposed dynamical mechanism. Geografiska Annaler: A, Physical Geography, 97 (1): pp.41-59.

Holdridge, R. (1982). Ecología basada en zonas de vida. Instituto Interamericano de Cooperación para la Agricultura. San José, Costa Rica. 216 p.

Jaramillo. (2005). Clima Andino y café colombiano. CENICAFE. P. 196.

Jiménez L. (2021). Inundaciones en Sixaola: “A pesar de que salió el sol, el agua sigue entrando”. Hay 279 personas en cuatro albergues ubicados en Matina, Talamanca y Valle de la Estrella. Teletica, Costa Rica.

Iizumi, T. et. al. (2014). Impacts of El Niño Southern Oscillation on the global yields of major crops. Nature communications, 5(1): 1-7.

Instituto de Hidrología, Meteorología y Estudios Ambientales. (2014). Determinación de un rango normal para la precipitación para la precipitación. Análisis comparativo entre los umbrales de normalidad. IDEAM. Instituto de Hidrología y Meteorología. Santa Fe de Bogotá, Colombia.

Mendizábal, M. (1973). Distribución de la precipitación con la altura. Tesis de Licenciatura, Universidad de Costa Rica, Sede Rodrigo Facio, San José.

McPhaden, M. (2006). Genesis and Evolution of the 1997-98 El Niño. Sciences, 314(5806): pp. 1740-1745. 
Ochoa, A. et. al. (2016). Evaluation of downscaled estimates of monthly temperature and precipitation for a Southern Ecuador case study. International Journal Climatology 36: pp. 1244-1255.

Organización Meteorológica Mundial. 2021. El Niño/La Niña Hoy Febrero de 2021. OMN. https:// public.wmo.int/es/el-ni\%C3\%B1ola-ni\%C3\%B1a-hoy. Revisado el 19 Mayo, 2021.

Peterson. T.C. et. al. (2002): Recent changes in climate extremes in the Caribbean region. Journal of Geophysical Research: Atmospheres, 107(D21), 16 pp.

Poveda. G., Jaramillo, L. and Vallejo, L.F. (2014): Seasonal precipitation patterns along pathways of South American low-level jets and aerial rivers. Water Resources Research, 50: pp. 98-118.

Quesada M and P. Waylen. (2020). Variability of Daily Precipitation on the Caribbean Coast of Costa Rica. Revista de Climatología. España.

Roy, S. and Balling Jr, R.C., 2004. Trends in extreme daily precipitation indices in India. International Journal of Climatology: A Journal of the Royal Meteorological Society, 24(4), pp.457-466.

SG-SICA. (2020). Pronostican fenómeno meteorológico de La Niña y lluvias arriba de lo normal para los próximos meses del año en los países miembros del SICA. Jueves, 23 de julio de 2020

Published by: Secretaría General del Sistema de la Integración Centroamericana

Sohrabi, M.M., Ryu, J.H. and Alijani, B., (2013). Spatial and temporal analysis of climatic extremes in the mountainous regions of Iran. International Journal of Climate Change: Impacts $\mathcal{E}$ Responses, 4(4).

Schultz, D.M., Bracken, W.E. and Bosart, L.F., (1998). Planetary-and synoptic-scale signatures associated with Central American cold surges. Monthy Weather Review, 126 (1): pp. 5-27.

Solano, J y R. Villalobos (2016). Regiones y Subregiones climáticas de Costa Rica. Instituto Meteorológico Nacional. Gestión para el desarrollo.

Taylor, K. E., Stouffer, R. J., and Meehl, G. A. (2012). An overview of CMIP5 and the experiment design. Bulletin of the American Meteorological Society, 93 (4): pp. 485-498. https://doi.org/10.1175/ BAMS-D-11-00094.1

Wang, C (2007). Variability of the Caribbean low-level jet and its relations to climate. Climate Dynamics, 29: pp. 411-422.

Waylen, P.R., Caviedes, C.N. and Quesada, M.E., (1996). Interannual variability of monthly precipitation in Costa Rica. Journal of Climate, 9 (10): pp. 2606-2613.

Cómo citar este artículo:

Quesada Q, Marvin E. (2021). Influencia de una depresión intermontana y la altitud entre dos montañas en la diferenciación espacial en la precipitación en Costa Rica. Cuadernos de Geografía, 106, 49-70.

https://doi.org/10.7203/cguv.106.20368

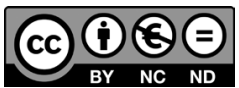

Este obra está bajo una licencia de Creative Commons Reconocimiento-NoComercial-SinObraDerivada 4.0 Internacional. 